\title{
Remarks on a conjecture about Randić index and graph radius
}

T. Dehghan-Zadeh, Hongbo Hua, A. R. Ashrafi, and N. Habibi 


\title{
REMARKS ON A CONJECTURE ABOUT RANDIĆ INDEX AND GRAPH RADIUS
}

\author{
T. DEHGHAN-ZADEH, HONGBO HUA, A. R. ASHRAFI, AND N. HABIBI
}

Received 5 December, 2012

\begin{abstract}
Let $G$ be a nontrivial connected graph. The radius $r(G)$ of $G$ is the minimum eccentricity among eccentricities of all vertices in $G$. The Randic index of $G$ is defined as $R(G)=$ $\sum_{u v \in E(G)} \frac{1}{\sqrt{d_{G}(u) d_{G}(v)}}$, and the Harmonic index is defined as $H(G)=\sum_{u v \in E(G)} \frac{2}{d_{G}(u)+d_{G}(v)}$, where $d_{G}(x)$ is the degree of the vertex $x$ in $G$. In 1988, Fajtlowicz conjectured that for any connected graph $G, R(G) \geq r(G)-1$. This conjecture remains still open so far. More recently, Deng et al. proved that this conjecture is true for connected graphs with cyclomatic number no more than 4 by means of Harmonic index. In this short paper, we use a class of composite graphs to construct infinite classes of connected graphs, with cyclomatic number greater than 4 , for which the above conjecture holds. In particular, for any given positive odd number $k \geq 7$, we construct a connected graph with cyclomatic number $k$ such that the above conjecture holds for this graph.
\end{abstract}

2010 Mathematics Subject Classification: 05C07; 05C12; 05 C76

Keywords: Randić index, radius, graffiti conjecture, cyclomatic number, harmonic index

\section{INTRODUCTION}

Let $G$ be a simple connected graph with vertex set $V(G)$ and edge set $E(G)$. For a graph $G$, we let $d_{G}(v)$ be the degree of a vertex $v$ in $G$ and $d_{G}(u, v)$ be the distance between two vertices $u$ and $v$ in $G$. Other notation and terminology not defined here will conform to those in [3].

Topological indices are numerical parameters of a graph which characterize the topological structure of the graph. Topological indices are usually graph invariants associated with a graph. As one of the most well-known and successful topological indices, Randić index, defined as $R(G)=\sum_{u v \in E(G)} \frac{1}{\sqrt{d_{G}(u) d_{G}(v)}}$, was introduced by Randić in [15]. Randić index gained much popularity during the past decades. Since its appearance, tremendous attention has been focused on the upper and lower bounds of this index. Bollobás and Erdös [2] proved that the Randić index of a graph of order

Research was supported in part by Natural Science Foundation of the Higher Education Institutions of Jiangsu Province (No. 12KJB110001) and NNSF of China (No. 11201227). 
$n$ without isolated vertices is bounded from below by $\sqrt{n-1}$; they left as an open problem to determine the minimum value of the Randic index for a graph $G$ with given minimum degree $\delta(G)$. Delorme et al. [5] gave a partial solution to this open problem for the case of $\delta(G)=2$. Furthermore, they completely solved this open problem for the case when $G$ is a triangle-free graph with given minimum degree $\delta(G)$. Balister et al. [1] determined the maximal Randić index of a tree with a given number of vertices and leaves. About reviews of mathematical properties of the Randic index, the interested readers are referred to [12,13]. Fajtlowicz [10] and Caporossi and Hansen [4] conjectured that the Randić index can be bounded from below in terms of the graph radius. In [8], Deng et al. gave a partial solution to this conjecture by studying the relationship between the Harmonic index and graph radius.

The Harmonic index of a connected graph $G$ is defined by Fajtlowicz [9] as $H(G)=\sum_{u v \in E(G)} \frac{2}{d_{G}(u)+d_{G}(v)}$. Favaron et al. [11] considered the relationship between the Harmonic index and graph eigenvalues. Zhong [17] determined the minimum and maximum values of the Harmonic index for simple connected graphs and trees, and characterized the corresponding extremal graphs. Deng et al. [6] considered the relationship between the Harmonic index $H(G)$ and the chromatic number $\chi(G)$ and proved that $\chi(G) \leq 2 H(G)$. This result strengthens a conjecture about the Randić index and the chromatic number which is based on the system AutoGraphiX. Deng et al. [7] gave a best possible lower bound for the Harmonic index of a graph and a triangle-free graph with minimum degree no less than two and characterized the corresponding extremal graphs, respectively.

In [10], Fajtlowicz proposed a conjecture concerning the relationship between the Randić index and graph radius, which reads as follows.

Conjecture 1 ([10]). For any connected $G, R(G) \geq r(G)-1$.

Caporossi and Hansen [4] partially proved this conjecture by showing that $R(T) \geq$ $r(T)+\sqrt{2}-\frac{3}{2}$ for any tree $T$. Liu and Gutman [14], and You and Liu [16] proved that the conjecture is true for unicyclic, bicyclic and tricyclic graphs.

In [8], Deng et al. proved the following result.

Theorem 1 ([8]). Let $G$ be a graph with cyclomatic number $k \geq 1$. Then $H(G) \geq$ $r(G)-\frac{31}{105}(k-1)$. In particular, $H(G)>r(G)-1$ for a graph with cyclomatic number no more than 4.

Remark 1 . For any connected graph $G$, since

$$
R(G)=\sum_{u v \in E(G)} \frac{1}{\sqrt{d_{G}(u) d_{G}(v)}} \geq \sum_{u v \in E(G)} \frac{2}{d_{G}(u)+d_{G}(v)}=H(G)
$$


and by Theorem $1, H(G)>r(G)-1$ for a connected graph $G$ with cyclomatic number no more than 4 , Conjecture 1 holds for those connected graphs $G$ with cyclomatic number no more than 4 .

In this short paper, we use a class of composite graphs to construct infinite classes of connected graphs, with cyclomatic number greater than 4 , for which Conjecture 1 holds. In particular, for any given positive odd number $k \geq 7$, we can construct a connected graph with cyclomatic number being $k$ such that the above conjecture holds for this graph. Consequently, our results extend those of Deng et al. in [6].

\section{MAIN RESULTS}

We first introduce a class of composite graphs, with which we are able to construct our desired graphs for which Conjecture 1 holds. The double graph $G^{*}$ of a given graph $G$ is constructed by making two copies of (including the initial edge set of each) and adding edges $u_{1} v_{2}$ and $u_{2} v_{1}$ for every edge $u v$ of $G$.

For each vertex $u$ in $G$, we call the corresponding vertices $u_{1}$ and $u_{2}$, in $G^{*}$, the clone vertices of $u$. If an $n$-vertex connected graph $G$ has a vertex of degree $n-1$, then $G$ is said to be well-connected.

Concerning the Harmonic index and radius of double graphs, we have the following result.

Theorem 2. Let $G$ be a nontrivial connected graph of order $n$, and let $G^{*}$ be its double graph. We have

(a) If $G$ is not well-connected, then $r\left(G^{*}\right)=r(G)$, and if $G$ is well-connected, then $r\left(G^{*}\right)=r(G)+1$;

(b) $H\left(G^{*}\right)=2 H(G)$.

Proof. It can be easily seen that if $G$ is connected, then $G^{*}$ is also connected. For the sake of convenience, we label all vertices of $G$ as $v_{1}, \ldots, v_{n}$. Suppose that $x_{i}$ and $y_{i}$ are the corresponding clone vertices, in $G^{*}$, of $v_{i}$ for each $i=1, \ldots, n$. Given a vertex $v_{i}$ in $G$. According to the definition of double graph, for any vertex $v_{j}$, different from $v_{i}$, in $G$, we have

$$
d_{G^{*}}\left(x_{i}, x_{j}\right)=d_{G^{*}}\left(x_{i}, y_{j}\right)=d_{G^{*}}\left(y_{i}, x_{j}\right)=d_{G^{*}}\left(y_{i}, y_{j}\right)=d_{G}\left(v_{i}, v_{j}\right) .
$$

Moreover, we have $d_{G^{*}}\left(x_{i}\right)=d_{G^{*}}\left(y_{i}\right)=2 d_{G}\left(v_{i}\right)$ for $i=1, \ldots, n$. Furthermore, we have

$$
d_{G^{*}}\left(x_{i}, y_{i}\right)=2
$$

for $i=1, \ldots, n$, since there exists at least one vertex, say $x_{k}$ (or $y_{k}$ ), such that both $x_{i}$ and $y_{i}$ are adjacent to $x_{k}$ (or $y_{k}$ ).

If $G$ is not well-connected, we have $e c_{G}\left(x_{i}\right) \geq 2$ for $i=1, \ldots, n$, that is, $r(G) \geq$ 2. Combining this fact and Eq.s (2.1) and (2.2), we have $e c_{G^{*}}\left(x_{i}\right)=e c_{G}\left(v_{i}\right)=$ $e c_{G^{*}}\left(y_{i}\right)$ for any $i=1, \ldots, n$, i.e., $r(G)=r\left(G^{*}\right)$. If $G$ is well-connected, then $G$ has a vertex, say $v_{i}$, of degree $n-1$. Thus, we have $e c_{G}\left(v_{i}\right)=1$ and $r(G)=1$. By 
Eq. (2.2), we have $r\left(G^{*}\right)=e c_{G^{*}}\left(x_{i}\right)=d_{G^{*}}\left(x_{i}, y_{i}\right)=2=r(G)+1$. This proves (a).

Now, we prove $(b)$. Let $h_{G}(u v)=\frac{2}{d_{G}(u)+d_{G}(v)}$. Then $H(G)=\sum_{u v \in E(G)} h_{G}(u v)$. For $1 \leq i, j \leq n$ and $i \neq j$,

$$
h_{G^{*}}\left(x_{i} x_{j}\right)=\frac{2}{d_{G^{*}}\left(x_{i}\right)+d_{G^{*}}\left(x_{j}\right)}=\frac{1}{d_{G}\left(v_{i}\right)+d_{G}\left(v_{j}\right)}=\frac{1}{2} h_{G}\left(v_{i} v_{j}\right) .
$$

By symmetry, we have

$$
h_{G^{*}}\left(x_{i} x_{j}\right)=h_{G^{*}}\left(x_{i} y_{j}\right)=h_{G^{*}}\left(y_{i} x_{j}\right)=h_{G^{*}}\left(y_{i} y_{j}\right)
$$

for each $i, j=1, \ldots, n$ and $i \neq j$. By means of Eq.s (2.3) and (2.4),

$$
\begin{aligned}
H\left(G^{*}\right) & =4 \sum_{x_{i} x_{j} \in E\left(G^{*}\right)} h_{G^{*}}\left(x_{i} x_{j}\right) \\
& =4 \sum_{v_{i} v_{j} \in E(G)} \frac{1}{2} h_{G}\left(v_{i} v_{j}\right) \\
& =2 H(G),
\end{aligned}
$$

which proves $(b)$.

We use Theorem 2 to prove the following consequence.

Theorem 3. There exist infinite class of connected graphs, with cyclomatic number greater than 4, for which Conjecture 1 holds. In particular, for any given positive odd number $k \geq 7$, there exists a connected graph with cyclomatic number being $k$ such that Conjecture 1 holds for this graph.

Proof. We first take a connected graph $G$ of order $n \geq 4$ and size $m$ such that it is not well-connected and its cyclomatic number is less than or equal to 4 . By Theorem $1, H(G)>r(G)-1$.

Now, we construct the double graph $G^{*}$ of $G$. Since $G$ is not well-connected, then by Theorem $2, H\left(G^{*}\right)=2 H(G)>2(r(G)-1)=2 r\left(G^{*}\right)-2>r\left(G^{*}\right)-1$, as $r\left(G^{*}\right) \geq 2$. Thus, $R\left(G^{*}\right) \geq H\left(G^{*}\right)>r\left(G^{*}\right)-1$. Moreover, we can find that the cyclomatic number of $G^{*}$ is $\left|E\left(G^{*}\right)\right|-\left|V\left(G^{*}\right)\right|+1=4 m-2 n+1 \geq 4(n-1)-$ $2 n+1=2 n-3 \geq 5$ for $n \geq 4$.

For a graph $G$, we now define its $k$-th iterated double graph $G^{k *}$ as

$$
G^{1 *}=G^{*} \text { and } G^{k *}=\left(G^{(k-1) *}\right)^{*} \text { for } k \geq 1
$$

and $G^{0 *}=G$ for consistence.

Note that $G^{*}$ is also not well-connected. Repeatedly using Theorem 2, we can prove that

$$
R\left(G^{k *}\right) \geq H\left(G^{k *}\right)=2^{k} H(G)>2^{k}(r(G)-1)=2^{k}\left(r\left(G^{k *}\right)-1\right)>r\left(G^{k *}\right)-1 .
$$


Similarly, the cyclomatic number of $G^{k *}$ is $\left|E\left(G^{k *}\right)\right|-\left|V\left(G^{k *}\right)\right|+1=4^{k} m-$ $2^{k} n+1 \geq 4^{k}(n-1)-2^{k} n+1=2^{k}\left(2^{k}-1\right) n-4^{k}+1=2^{k}\left[2^{k}(n-1)-n\right]+1 \geq$ $2 n-3 \geq 5$ for $k \geq 1$ and $n \geq 4$.

Now, for a given positive odd number $k=2 k^{\prime}+1 \geq 7$, we construct a connected graph with cyclomatic number being $k$. In fact, we first take a graph $G$ with $k^{\prime}$ vertices and $k^{\prime}$ edges. Let $G^{*}$ be the double graph of $G$. Then the cyclomatic number of $G^{*}$ is equal to $\left|E\left(G^{*}\right)\right|-\left|V\left(G^{*}\right)\right|+1=2 k^{\prime}+1=k$. By the previous proof, we know that $R\left(G^{*}\right) \geq H\left(G^{*}\right)>r\left(G^{*}\right)-1$. This completes the proof.

\section{REFERENCES}

[1] P. Balister, B. Bollobás, and S. Gerke, "The generalized Randić index of trees," J. Graph Theory, vol. 56, no. 4, pp. 270-286, 2007.

[2] B. Bollobás and P. Erdős, "Graphs of extremal weights," Ars Comb., vol. 50, pp. 225-233, 1998.

[3] J. A. Bondy and U. S. R. Murty, Graph theory with applications. New York: American Elsevier Publishing Co., 1976.

[4] G. Caporossi and P. Hansen, "Variable neighborhood search for extremal graphs. I: The AutoGraphiX system," Discrete Math., vol. 212, no. 1-2, pp. 29-44, 2000.

[5] C. Delorme, O. Favaron, and D. Rautenbach, "On the Randić index," Discrete Math., vol. 257, no. 1, pp. 29-38, 2002.

[6] H. Deng, S. Balachandran, S. K. Ayyaswamy, and Y. B. Venkatakrishnan, "On the harmonic index and the chromatic number of a graph," preprint.

[7] H. Deng, Z. Tang, and R. Wu, "A lower bound for the harmonic index of a graph with minimum degree at least two," preprint.

[8] H. Deng, Z. Tang, and J. Zhang, "On a conjecture of Randić index and graph radius," available online at: http://arxiv.org/pdf/1210.2543.pdf.

[9] S. Fajtlowicz, "On conjectures of Graffiti. II," in Combinatorics, graph theory, and computing, Proc. 18th Southeast. Conf., Boca Raton/Fl. 1987, Congr. Numerantium, 1987, vol. 60, pp. 189197.

[10] S. Fajtlowicz, "On conjectures of Graffiti," Discrete Math., vol. 72, no. 1-3, pp. 113-118, 1988.

[11] O. Favaron, M. Mahio, and J. F. Saclé, "Some eigenvalue properties in graphs (Conjectures of Graffiti-II)," Discrete Math., vol. 111, pp. 197-220, 1993.

[12] I. Gutman and B. Furtula, Eds., Recent Results in the Theory of Randic Index, ser. Mathematical Chemistry Monograph No. 6. Kragujevac, 2008.

[13] X. Li and I. Gutman, Mathematical Aspects of Randić-type Molecular Structure Descriptors, ser. Mathematical Chemistry Monographs No. 1. Kragujevac, 2006.

[14] B. Liu and I. Gutman, "On a conjecture on Randić indices," MATCH Commun. Math. Comput. Chem., vol. 62, no. 1, pp. 143-154, 2009.

[15] M. Randić, "Characterization of molecular branching," J. Am. Chem. Soc., vol. 97, pp. 6609-6615, 1975.

[16] Z. You and B. Liu, "On a conjecture of the Randić index," Discrete Appl. Math., vol. 157, no. 8, pp. 1766-1772, 2009.

[17] L. Zhong, "The harmonic index for graphs," Appl. Math. Lett., vol. 25, no. 3, pp. 561-566, 2012. 
Authors' addresses

T. Dehghan-Zadeh

Department of Pure Mathematics, Faculty of Mathematical Science,, University of Kashan, Kashan 87317-51167, I. R. Iran

\section{Hongbo Hua}

Huaiyin Institute of Technology, Faculty of Mathematics and Physics, 223003 Huai'an City, PR China

E-mail address: hongbo. hua@gmail.com

\section{A. R. Ashrafi}

Department of Pure Mathematics, Faculty of Mathematical Science,, University of Kashan, Kashan 87317-51167, I. R. Iran

E-mail address: ashrafi@kashanu.ac.ir

N. Habibi

Department of Mathematics, Faculty of Science,, University of Zanjan, Zanjan, I. R. Iran 